\title{
The Effects of Ultra-early Hyperbaric Oxygen Intervention in the Treatment of Diffuse Axonal Injury
}

\author{
Xinli Feng1, Meng Sun ${ }^{2 *}$ \\ ${ }^{1}$ Hyperbaric Oxygen Treatment Room, Hebei University Hospital, Baoding 071000, Hebei Province, China \\ 2Interventional Vascular Surgery, Hebei University Hospital, Baoding 071000, Hebei Province, China \\ *Corresponding author: Meng Sun, sunmeng135135@sina.com
}

\begin{abstract}
Objective: To investigate the efficacy and mechanism of ultra-early hyperbaric oxygen intervention in the treatment of diffuse axonal injury (DAI). Methods: Eighty-six patients with diffuse axonal injury were selected and then divided into an ultra-early hyperbaric oxygen treatment group and a conventional treatment group with 43 patients in each group. The Glasgow Coma Scale (GCS) on the $10^{\text {th }}$ day (10d), $20^{\text {th }}$ day (20d), and $30^{\text {th }}$ day (30d) after treatment and the Glasgow Outcome Score (GOS) 6 months later were observed and compared between both the groups. Results: The average score of the GCS at $10 \mathrm{~d}, 20 \mathrm{~d}$, and $30 \mathrm{~d}$ as well as the GOS 6 months later in the ultra-early hyperbaric oxygen treatment group were higher than those in the conventional treatment group $(\mathrm{P}<0.05)$. Conclusion: Hyperbaric oxygen therapy is one of the unique and effective methods in clinical treatment especially for the treatment of DAI patients and it is worthy of promotion.
\end{abstract}

Keywords: Diffuse axonal injury; Hyperbaric oxygen therapy; Ultra-early

Publication date: July 2021; Online publication: July 31, 2021

\section{Introduction}

Diffuse axonal injury (DAI) is a brain injury caused by acceleration and/or deceleration of the head. It is usually due to a blunt force on the head that causes diffuse damage to the brain's axonal proteins. This is characterized by extensive axonal rupture which is common in injuries from car accidents and falls. Consciousness is a typical clinical manifestation in which the condition of the affected patient would be critically ill. Both the clinical disability rate and fatality rate are high which account for about $35 \%$ in addition to a poor prognosis ${ }^{[1-2]}$. At present, there is no effective treatment method for DAI. In recent years, the widespread use of hyperbaric oxygen therapy provides an effective treatment approach in DAI therapy. It has been clinically verified that hyperbaric oxygen therapy has a good effect in the recovery of brain injury, cerebral ischemia, and stroke ${ }^{[3-4]}$. In order to improve the understanding of the disease and the treatment effects, the data of 86 patients with DAI have been analyzed.

\section{Materials and methods}

\subsection{Clinical data}

A total of 86 patients with diffuse axonal injury admitted to Hebei University Hospital from February 2018 to February 2020 were selected. There were 48 male patients and 38 female patients, aging from 13 to 61 years old, admitted within 1 hour to 24 hours after injury with GCS $<8$, diagnosed as DAI by computed tomography (CT) scan after admission with clinical manifestations. The 86 patients were divided into 2 
groups in which 43 of them were in the ultra-early hyperbaric oxygen treatment group and the other 43 in the conventional treatment group. The two groups were not statistically significant in terms of age, gender, course of disease, as well as complications ( $\mathrm{P}>0.05)$, and they were comparable.

\subsection{Treatment}

Both groups of patients were given dehydration, anti-infection, hormones, and consciousness-regaining treatment while their airways were kept unobstructed, and tracheotomy was done if necessary. In addition to the above comprehensive treatment, hyperbaric oxygen treatment was started within 24 to 72 hours in the ultra-early hyperbaric oxygen treatment group. The YC2470/0.3-101V multi-person air pressurized chamber was used. The treatment pressure was at 1.8 ATA and pressurized for 20 minutes, and then stabilized. Oxygen was given for 60 minutes and decompression for 20 minutes once a day. Each course of treatment lasted 10 times and between each course, 3 rest-days were provided.

\subsection{Efficacy evaluation}

The Glasgow scores of the ultra-early hyperbaric oxygen treatment group and the control group were compared and analyzed. The Glasgow Coma Scale (GCS) was used for each group of patients at 10d, 20d, and 30d after treatment while the Glasgow Outcome Score (GOS) was used after 6 months of treatment. The criteria for Glasgow Outcome Score: (1) Good recovery: 5 points; (2) Moderate disability: 4 points; (3) Severe disability: 3 points; (4) Vegetative state: 2 points; (5) Death: 1 point.

\subsection{Statistical analysis}

Statistical Package for the Social Sciences (SPSS) version 17.0 software was used to analyze the data. The measured data were tested by t-test and the count data were tested by chi-square $\left(\mathrm{X}^{2}\right)$. The data was represented by $(\mathrm{x} \pm \mathrm{s})$ and the differences were statistically significant when $\mathrm{P}<0.05$.

\section{Results}

\subsection{Comparison of GCS between the two groups of patients}

The ultra-early hyperbaric oxygen treatment group had higher GCS at 10d, 20d, and 30d which was significantly different from the conventional treatment group $(\mathrm{P}<0.05)$. See Table 1.

Table 1. Comparison of GCS between the two groups after treatment

\begin{tabular}{ccc}
\hline \multirow{2}{*}{ Evaluation time } & \multicolumn{2}{c}{ GCS (points) } \\
\cline { 2 - 3 } & $\begin{array}{c}\text { Conventional treatment } \\
\text { group }\end{array}$ & $\begin{array}{c}\text { Ultra-early hyperbaric oxygen treatment } \\
\text { group }\end{array}$ \\
\hline 10d & $6.68 \pm 2.01$ & $7.88 \pm 3.02$ \\
20d & $8.32 \pm 2.31$ & $10.12 \pm 3.68$ \\
30d & $11.52 \pm 3.16$ & $13.86 \pm 2.56$ \\
\hline
\end{tabular}

Note: Comparing with the conventional treatment group, $\mathrm{P}<0.05$.

\subsection{Comparison of the prognosis of the two groups of patients using GOS}

The patients in the ultra-early hyperbaric oxygen treatment group had better prognosis than the conventional treatment group $(\mathrm{P}<0.05)$. See Table 2. 
Table 2. Comparison of prognostic assessment with GOS between the two groups of patients after 6 months of treatment

\begin{tabular}{cccc}
\hline Group & Number of cases & Good prognosis & Poor prognosis \\
\hline $\begin{array}{c}\text { Conventional treatment } \\
\text { group }\end{array}$ & 43 & $16(37.2 \%)$ & $27(62.8 \%)$ \\
$\begin{array}{c}\text { Ultra-early hyperbaric } \\
\text { oxygen treatment group }\end{array}$ & 43 & $28(65.1 \%)$ & $15(34.2 \%)$ \\
\hline
\end{tabular}

Note: Comparing with the conventional treatment group, $\mathrm{P}<0.05$.

\section{Discussion}

Diffuse axonal injury (DAI) is the injury of axons and capillaries in many parts of the brain tissue due to the shearing force when the head is subjected to linear or rotational acceleration. It is common in traffic accidents, falls from high altitudes, or intense violence. Clinically, if diffuse axonal injury is not diagnosed and treated in time, it may further develop into raised intracranial pressure, aggravate brain tissue lesions, and eventually lead to brain herniation, or even death from central nervous system failure ${ }^{[5-6]}$. At present, the treatment of cerebral edema and the control of secondary brain injury are clinically regarded as the key to the treatment of diffuse axonal injury. Most of them focus on the treatment of cerebral edema, reducing intracranial pressure, preventing secondary brain damage, and other comprehensive treatments. These treatment measures have not significantly improved the prognosis of patients ${ }^{[7-8]}$.

Hyperbaric oxygen therapy is an adjuvant therapy that has rapidly developed in recent years. Currently, it is mainly used in the treatment of hypoxic ischemic diseases and brain trauma. Hyperbaric oxygen treatment for DAI can increase blood oxygen concentration, oxygen diffusion between tissues, and improve cerebral hypoxia ${ }^{[9-10]}$. Patients with DAI often have extensive swelling of the cerebral hemispheres, expansion and congestion of cerebral blood vessels, and a significant increase in cerebral blood flow as well as the brain stem volume. Hyperbaric oxygen therapy can constrict cerebral blood vessels and reduce cerebral blood flow, thereby reducing cerebral edema ${ }^{[11]}$. Hyperbaric oxygen improves cerebral vasoconstriction and reduces cerebral blood flow, vascular permeability, exudation, cerebral edema, as well as intracranial pressure accordingly. While hyperbaric oxygen reduces cerebral blood flow, it increases blood flow in the vertebral arteries. It also improves the hypoxic state of the reticular ascending activation system and increases the brainstem oxygen partial pressure while helping to improve the state of wakefulness and promote the regain of consciousness ${ }^{[12-13]}$. Clinical studies have shown that the use of hyperbaric oxygen therapy as soon as possible after the occurrence of DAI can effectively reduce the mortality rate and improve the prognosis of patients ${ }^{[14-15]}$.

The 43 DAI patients in this group started hyperbaric oxygen therapy within 24 to 72 hours of their injuries. The average change of their GCS on the $10^{\text {th }}, 20^{\text {th }}$, and $30^{\text {th }}$ day after treatment as well as their GOS after 6 months were significantly higher than those in the conventional treatment group. GCS reflects the changes in patients' post-injury consciousness. The scores of the hyperbaric oxygen treatment group were higher than that of the conventional treatment group indicating that hyperbaric oxygen treatment has a certain effect in promoting patients' recovery and the recovery of their brain functions. Secondary injury after DAI is an important factor affecting the prognosis of patients. Early hyperbaric oxygen therapy after the occurrence of DAI can reduce common complications of craniocerebral injury and patient mortality in addition to enhance their quality of life.

In summary, early adjuvant treatment of hyperbaric oxygen for patients with diffuse axonal injury would help repair injured axons, avoid aggravation of secondary brain injury, restore nerve functions, and 
significantly improve their prognosis. This has an important clinical application value and is worthy of promotion.

\section{Disclosure statement}

The author declares no conflict of interest.

\section{References}

[1] Li G, Yuan S, Xiao W, et al., 2016, Early Hyperbaric Oxygen Treatment of 30 Patients with Diffuse Axonal Injury. Hainan Medicine, 27(2): 287-8.

[2] Cui Y, Liu Z, Jiang S, et al., 2019, Efficacy of Hyperbaric Oxygen Combined with Gangliosides in the Treatment of Diffuse Axonal Injury and its Influence on Serum Related Indicators and Prognosis. Chinese Journal of Nautical Medicine and Hyperbaric Medicine, 26(5): 423-6.

[3] Cardenas DP, Muir ER, Huang S, 2015, Functional MRI During Hyperbaric Oxygen: Effects of Oxygen on Neurovascular Coupling and Bold FMRI Signals. Neuroimage, 15(2): 212-3.

[4] Hou G, Liu J, Wang C, et al., 2016, Quantitative EEG Relative Power Changes and Their Significance in Patients with Diffuse Axonal Injury Treated with Hyperbaric Oxygen at Different Pressures. Shandong Medicine, 56 (43): 79-81.

[5] Yang Y, Zhang YG, Lin GA, et al., 2014, The Effects of Different Hyperbaric Oxygen Manipulations in Rats After Traumatic Brain Injury. Neurosci Lett, 563(5): 38-43.

[6] Reynolds CA, Kallakuri S, Bagchi M, et al., 2011, Endothelin Receptor an Antagonism Reduces the Extent of Diffuse Axonal Injury in a Rodent Model of Traumatic Brain Injury. Neurolog Res, 33(2): 192-6.

[7] Harch PG, 2015, Hyperbaric oxygen in chronic traumatic brain injury: Oxygen, pressure, and gene therapy. Med Gas Res, 14(1): 5.

[8] Yang Y, Mei H, Liu J, et al., 2015, The Effect of Hyperbaric Oxygen Therapy at Different Pressures on the Prognosis of Severe Diffuse Axonal Injury. Chinese Journal of Nautical Medicine and Hyperbaric Medicine, 22(5): 378-81.

[9] Dietrich WD, Bramlett HM, 2010, The Evidence for Hypothermia as A Neuroprotectant in Traumatic Brain Injury. Neurotherapeutics, 7(1): 43-50.

[10] Zhou S, 2012, The Long-Term Effect of Hyperbaric Oxygen in the Treatment of Severe Head Injury. Jiangsu Medicine, 38(19): 2273-5.

[11] Wu C, Wang L, Yao J, 2012, Early Hyperbaric Oxygen Treatment of 45 Cases of Diffuse Axonal Injury of the Brain. Chinese Journal of Trauma, 28(1): 40-2.

[12] Gao C, Yang J, Zhai X, 2008, Basic and Clinical Medicine of Hyperbaric Oxygen, People's Medical Publishing House, Beijing, 189.

[13] Zhang Y, Wang X, Cheng P, 2015, Efficacy of Early Hyperbaric Oxygen in the Treatment of Diffuse Axonal Injury. Chinese Journal of Clinical Neurosurgery, 20(6): 366-7.

[14] Yan X, Mo Y, Huo G, et al., 2017, Analysis of the Clinical Efficacy of Hyperbaric Oxygen in the Treatment of Diffuse Axonal Injury. Western Medicine, 29(2): 222-5, 229.

[15] Li M, Wei J, et al., 2013, The Pathogenesis and Treatment Progress of Diffuse Axonal Injury. Chinese Medical Journal, 93(5): 397-9. 